Radiologe $2009 \cdot 49: 7$

DOI 10.1007/s00117-008-1788-5

Online publiziert: 22. November 2008

๑) Springer Medizin Verlag 2008

\section{Czerny ${ }^{1} \cdot \mathrm{H}$. Imhof $^{2}$}

${ }^{1}$ Klinische Abteilung für Neuroradiologie und muskuloskelettale Radiologie, Universitätsklinik für Radiodiagnostik, Medizinische Universität, Wien

${ }^{2}$ Universitätsklinik für Radiodiagnostik, Medizinische Universität, Wien

\title{
Bildgebende Diagnostik des Pharynx und Larynx
}

\section{Anatomie - Pathologie}

Liebe Leserinnen und Leser,

die Schnittbilddiagnostik im Kopf-HalsBereich hat in den letzten Jahrzehnten einen immer größeren Stellenwert eingenommen. Neue diagnostische Verfahren wie die Positronenemissionstomographie (PET) sowie die Kombination aus Positionemissionstomographie und Computertomographie (PET-CT) spielen eine wichtige Rolle zur Abklärung blastomatöser Prozesse der Kopf-Hals-Region. Die bildgebende Diagnostik, mit der die genaue Ausdehnung des Primärtumors bestimmt wird, spielt neben dem klinischen Staging und der Panendoskopie eine entscheidende Rolle bei der Therapie. Insbesondere die PET und die PET-CT haben zusätzlich noch das Abklärungsspektrum erweitert, da durch diese Verfahren oft noch weitere tumoröse Begleiterkrankungen nachgewiesen oder ausgeschlossen werden können.

Im vorliegenden Themenheft werden daher zunächst die Normalanatomie der pharyngealen und laryngealen Strukturen mittels der Computertomographie (CT) und Magnetresonanztomographie (MRT) beschrieben. Im Anschluss daran werden die pathologischen Prozesse des Pharynx und Larynx besprochen. Der Pharynx wird hierbei von 3 namhaften Autoren abgehandelt und in seine anatomischen und auch therapeutisch wichtigen Abschnitte, wie den Epi- oder Nasopharynx, den Oropharynx und Hypopharynx, eingeteilt und dargestellt.
Auch der Larynx und seine Pathologien werden von einer bekannten Autorin beschrieben.

Da aber den Radiologen mit der CT und MRT nicht nur präoperative und prätherapeutische Situationen begegnen, werden auch die posttherapeutischen und postoperativen Veränderungen des Pharynx und Larynx erläutert.

In der heutigen Zeit werden von den Radiologen besondere Kenntnisse der computer-, magnetresonanz- und auch positronenemissionstomographischen Darstellung und Bildinterpretation bzgl. der Anatomie und Pathologie von Pharynx und Larynx erwartet.

Wir hoffen daher, Ihnen mit dem folgenden Heft eine Hilfestellung geben zu können.

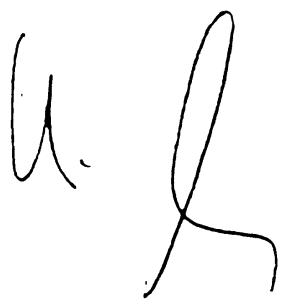

Univ.-Prof. Dr. Christian Czerny

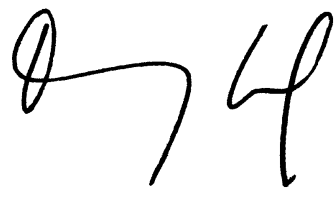

Univ.-Prof. Dr. Herwig Imhof 